Journal of Jazz Studies vol. 8, no. 2, pp. 114-136 (Winter 2012)

\title{
Ordered Step Motives in Jazz Standards
}

\author{
Keith Salley
}

\section{INTRODUCTION}

In listening to or performing jazz standards, I am frequently struck by the way linear motives help them cohere. I find it even more remarkable when these linear motives occur at various structural levels (e.g., across a single chord or a local chord progression, or across a phrase or a section of a composition). A number of publications have addressed short- and long-range linear connections in jazz compositions and improvisations by applying Schenkerian analytical techniques. ${ }^{1}$ Martin (1996) and Larson (1998 and 2009) have made especially persuasive, yet different arguments for such applications, demonstrating that the structure of jazz music-be it improvised or composed-has much in common with Western art music. While I certainly do not mean to dismiss this scholarship, I introduce a new analytical tool here-one that models linear relationships in a different way.

This analytical tool is especially useful in revealing linear connections across compositions that are tonally capricious or ambiguous. And in one case, it shows how long- and short-range motives lend continuity to a compelling circular form. ${ }^{2}$ Works that span a number of tonal centers, those with no clear global tonic, and those whose structures are not tonally closed do not easily find explication through Schenkerian methods. ${ }^{3}$ In some ways, this is due to the limitations of the monotonal orientation inherent to a Schenkerian perspective. Some jazz compositions demonstrate remarkable degrees of motivic coherence-locally and globally-when their melodies are not

\footnotetext{
${ }^{1}$ See, for example Larson $(1993,1998,1999,2005,2009)$, and also Strunk $(1985,1996)$, and Martin (1996, 2005, 2006, 2011).

${ }^{2}$ This study contributes to the written body of theoretical knowledge about jazz composition by engaging with current scholarship on tonal ambiguity, as in Strunk (2002), circular form, as in Waters (2011) and Larson et al. (2008), and nonfunctional harmony, as in Julien (2001), Strunk (2005), and Waters (2005, and 2011). It also contributes to previous studies of step collections in jazz such as Pressing (1978) and Tymoczko (1997), though these studies are primarily concerned with improvisation rather than composition.

${ }^{3}$ See, however, Salzer (1962), Morgan (1976), and Baker (1980) for examples of attempts to model Schenkerian hearings of music that could be similarly described.
}

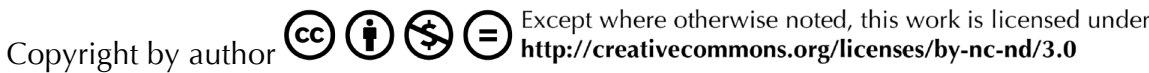


diatonically normalized, when tonal centers are not regarded as Stufen within a larger tonal framework, and even when no global tonic is emergent. With this article, I wish to create a space in our literature to showcase an alternative means of describing linear aspects of jazz compositions. ${ }^{4}$ In order to accommodate the types of works targeted here, this new descriptive space does not rely on a monotonal perspective.

To this end, I introduce an idea called the ordered step motive (OSM). While an OSM analysis does not attempt to contextualize distant tonalities within a single global collection, it may still reveal interesting things about key relationships. And as it does not normalize altered melodic tones, its stricter representation of intervallic space better represents the melodic and harmonic richness of standard jazz repertoire. OSMs often sound at musical surfaces, but they may also sound at deeper levels of structure. These latter occurrences are particularly relevant when they originate through linear intervallic patterns (LIPs), but also when they align - especially in patterned ways-with other OSMs. When OSMs do occur at multiple structural levels, they often reveal relationships between surface-level motives and underlying counterpoint. These relationships can cross multiple structural levels in compositions whose harmonic or melodic structures resist Schenkerian or neo-Schenkerian reductive approaches.

An OSM is an ordered ascending or descending stepwise collection of pitches that plays a significant motivic role in a piece. OSMs are expressed numerically in a way that indicates melodic direction and intervallic size. Numeric values correspond to the highness and lowness of each pitch, and the differences in value between numbers reflect distances in semitones between pitches. The step in the name "ordered step motive" reflects the rule that successive elements in an OSM can be no more than a whole tone apart. I have limited the OSM to segments with no changes of direction mainly for two reasons. First, a discussion including segments that change direction would

${ }^{4}$ For the analyses in this study I consulted the first commercial recordings of each composition. Fortunately, all but two of these also featured the composer as performer. As Jerome Kern's "All the Things You Are" (Example 6) was not written by a jazz musician, I consulted Gillespie (1945) - a seminal recording. Harmonic differences between Gillespie's version and Kern's original score from 1939 (notably at mm. 6, 14, and 24 of Example 6) are not crucial to my analysis. The case for Benny Golson's "Stablemates" (Example 5) is more complicated. The first two recorded performances are Davis (1955) and Chambers (1956), which predate Gillespie (1957), the first recording to feature the composer as performer. However, Gillespie (1957) is a big band performance. As such, it is a very arranged performance with a large number of incidental harmonies that would not reflect Golson's original conception of the tune in a general enough way to make my points about linear intervallic patterns. For this composition, I consulted Davis (1955). 
quickly exceed the scope of this article. But more importantly, such segments are characterized more strongly by their general contours than by their specific intervallic sizes. For this reason, analytical approaches to melodic contour are often more appropriate for modeling ordered segments with changes of melodic direction. ${ }^{5}$

As ordered segments, OSMs are subject to the same canon of operations that relate serial elements like tone rows and contour segments. ${ }^{6}$ In addition to transpositional relationships among segments with the same intervallic structures, we can consider equivalence among OSMs that relate by retrograde, inversion, and a combination of the two. Important connections between nonequivalent OSMs can involve subset/superset relationships. In these ways, the OSM lies at an intersection of tonal and post-tonal conceptual systems. While works from tonal art music may also feature backward- and inversionallyrelated motives and themes, strict preservation of intervallic patterning in this music is seldom attained. This is due, in part, to general stylistic preferences to adhere melodically to operative diatonic collections (i.e., to preserve the pattern of half steps and whole steps within the tonic scale), or to refrain from modulating too frequently. Jazz standards are not necessarily constrained by the same concerns. It is common for a single four- or eight-measure phrase to visit three or more keys, and melodies are often comprised of notes that fall outside of the diatonic collections that are created or implied by the harmonies that underlie them. The combination of these traits enables numerous possibilities for strict motivic patterning to arise, and OSMs provide a way to account for some of these correspondences.

To illustrate, Figure 1 shows a family of equivalent OSMs placed within the same pitch range for ease of comparison. OSM $<0234>$ refers to the melodic path of a whole step followed by two half steps, ascending. The retrograde is OSM <4 32 0 $>$, which represents two descents by semitone followed by a step of a whole tone. From this it follows that OSM $<4210>$ is the inversion, and the retrograde inversion is OSM $<01224>$. Motives that relate through any of the operations shown in Figure 1 are, in a sense, equivalent, and this study recognizes no other operations that generate equivalent OSMs.

\footnotetext{
${ }^{5}$ See, for example, Marvin and Laprade (1987), Morris (1987), and Friedmann (1985).

${ }^{6}$ Marvin and Laprade (1987) define contour segments.
} 
Figure 1: Canonic Operations on OSMs.

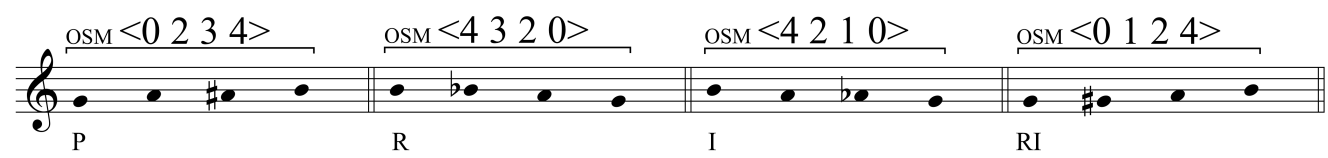

Many OSMs in the examples below arise through tonal processes. Some of these processes presuppose the hierarchical distinctions of Schenkerian analysis, and others do not. OSMs may sound in LIPs, in linear approaches to relatively stable resolutions (such as to the tonic of a II $\rightarrow \mathrm{V} \rightarrow$ I progression), and in conjunction with other OSMs that span larger segments. But they may also simply arise through conjunct melodic motion on the surface of a composition. With regard to the post-tonal aspect of OSM analysis, the canonic operations on OSMs are invaluable. By recognizing a family of OSMs that are equivalent at some level of abstraction, we can account precisely for relationships among motives with the same basic structure that may occur in different orientations. These orientations can reveal relationships between tonalities, and even general trends in standard jazz repertoire.

\section{ANALYSES}

My first analysis demonstrates some rather surprising correspondences among OSMs in Dave Brubeck's "It's A Raggy Waltz." While this composition is relatively unambiguous from a tonal perspective, it features every member of the family of OSMs from Figure 1. Example 1A shows relevant material from the A section. Here, OSM <0 23 4> sounds across mm. 1-3. Reciprocally,

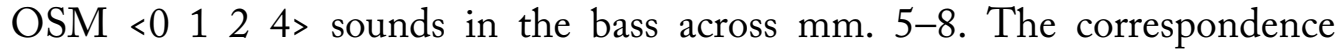
between these motives is clear, even though the second occurrence is actually a retrograde inversion of the first.

In the $\mathrm{A}$ section of Example 1, tonal processes clearly give the pitch elements of OSMs the salience that makes them motivic. Prolongations of tonic and subdominant harmonies create third progressions. At the same time, the chromatic passing tones within those third progressions play an important role in enabling us to hear how they relate. For this reason, the motive's label reflects its intervallic identity to the last detail. 
Example 1: Dave Brubeck, “It's A Raggy Waltz" (Brubeck 1961).
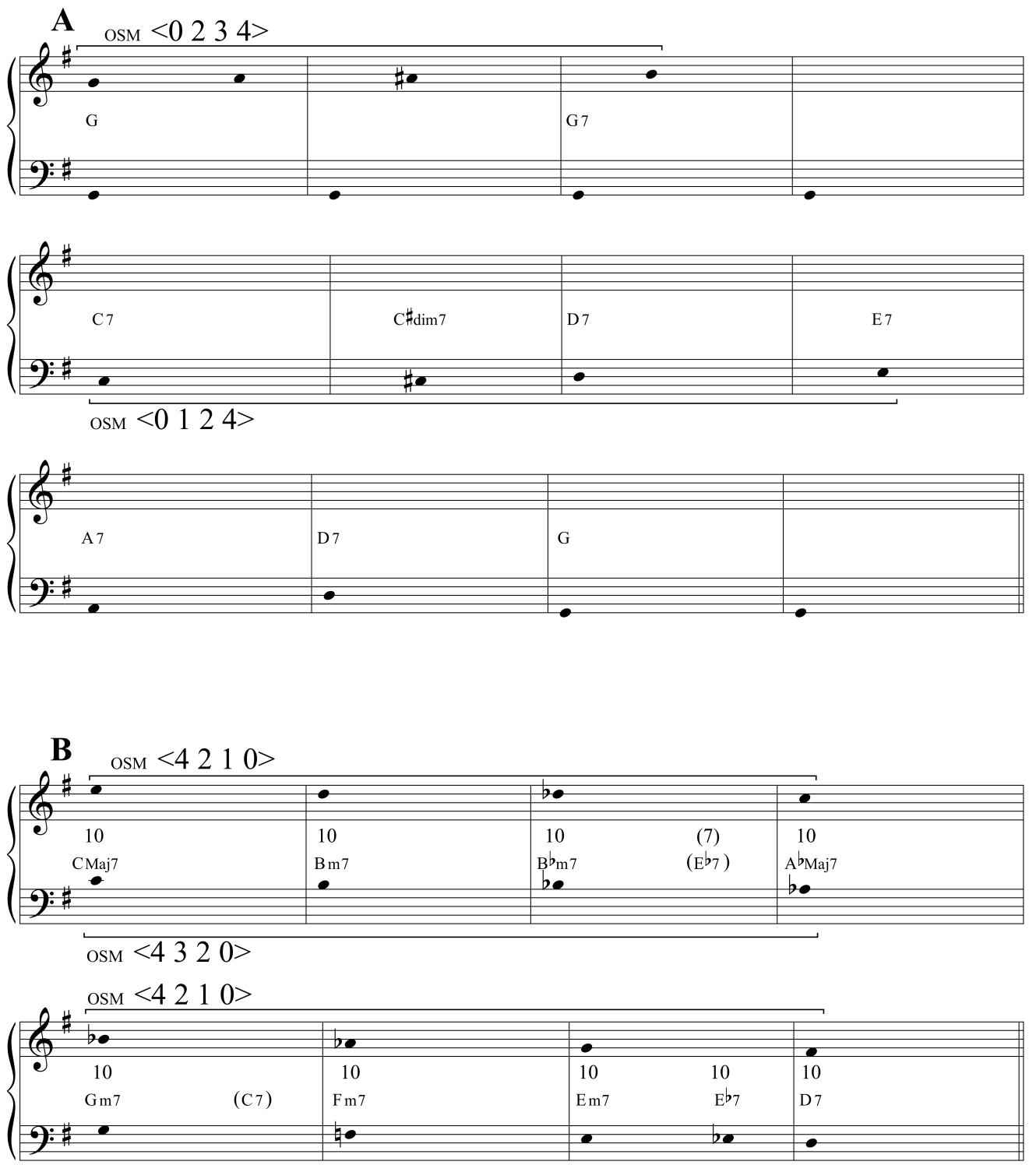

osm $<53210>$

While the A section of "It's A Raggy Waltz" does involve some secondary harmonies, it firmly establishes a single key by progressing through its tonicizations in a manner that resonates with conventional tonal movement in the key of G. On the other hand, the bridge (mm. 13-20) visits several keys. Here, tonicizations of $\mathrm{Ab}$ major and $\mathrm{F}$ minor and a strong arrival on $\mathrm{D} 7$ occur within eight measures. The overall melodic characteristics of both sections (not illustrated) are different, too. The A section is quite active. It establishes cross- 
rhythms and a compound melody. On the other hand, the melody in the B section is rhythmically simpler. Only one note sounds per chord and the texture is strictly monophonic. In spite of these differences, common OSMs help these sections relate. OSMs related to the opening motive sound across mm. 13-16 in parallel tenths against the bass. OSM $<4210>$, an inversion of the occurrence across mm. 1-3, sounds above OSM $<4320>$, a retrograde. Another inversionally-related OSM sounds in the melody across the second half of the bridge. This creates tenths above OSM $<53210>$, which is a superset of the inverted form of the motive. ${ }^{7}$ This motive only occurs because Brubeck composed a tritone substitute for $\mathrm{V} / \mathrm{V}$, opting for $\mathrm{Eb} 7$ instead of A7. For this reason, performers and composers should be sensitive to the possibility that harmonic substitutions may affect the motivic connections that unify a work.

These motivic recurrences do more than unify the work across the surface. They also help outline a functional harmonic progression on a larger scale. Example 2 shows how elements from OSMs in the upper voice create tenths against key tones in the bass, projecting the large-scale progression I $\rightarrow$ IV $\rightarrow$ $\mathrm{V} \rightarrow \mathrm{I}$ across the composition. In the A section, the opening OSM sounds over a tonic pedal and spans the root and third of the tonic chord. The stemmed notes across the $\mathrm{B}$ section are the first and last melodic tones. The bass notes in this section come from the first and last chords, IV7 and V7. The first OSM in the right hand spans the root and third of the subdominant chord, while the second drives toward the dominant and terminates on the leading tone. ${ }^{8}$

Example 2: Interaction of OSMs with large-scale harmonic progression in "It's A Raggy Waltz."

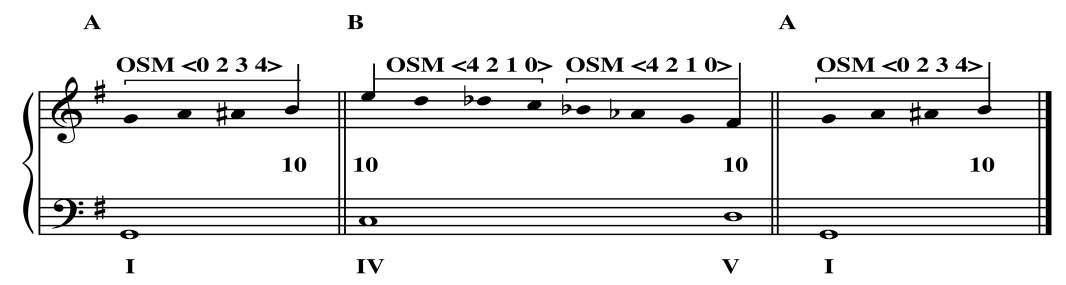

\footnotetext{
${ }^{7}$ In other words, OSM $<4210>$ is a subset of OSM $<53210>$. The first four elements of the latter constitute the former.

8 This observation is consistent with Larson (2003, 10), which compares the structural functions of musical bridges to actual, architecturally engineered bridges and observes how both tend to provide connectivity between structurally sound locations. Musical bridges often do this by beginning with a dominant-preparation chord and ending with a dominant chord.
} 
Examples 3-5 demonstrate how OSMs consisting of locally diatonic scale steps can relate passages that visit different keys. Example 3 shows patterns of related OSMs across the A section of Brubeck's "In Your Own Sweet Way." $\mathrm{OSM}<542$ 0 > sounds across mm. 1-2, accompanied by ii ${ }^{\varnothing} \rightarrow \mathrm{V} 7 \rightarrow$ i7 in $\mathrm{G}$ minor. OSM <5 310 p, related by retrograde inversion, sounds across the beginning of the second half, accompanied by ii7 $\rightarrow$ bII7 $\rightarrow$ I7 in Gb major. ${ }^{9}$ The inversional aspect of this relationship is a reflection of the fact that two diatonic collections related by ic 4 create a symmetrical pattern of steps around an inversional axis. Figure 2 illustrates this relationship, showing how the collections $\mathrm{G}$ minor (equivalent to $\mathrm{B} b$ major) and $\mathrm{G} b$ major align in this way around $\mathrm{B} b$. This member of the inversional axis is the global tonic of the composition, and it is also the point of arrival of both OSMs in the composition. ${ }^{10}$ The retrograde aspect of this motivic relationship allows both motives to share this common goal while descending, thereby making their correspondence all the more audible.

Example 3: Dave Brubeck, "In Your Own Sweet Way," A section (Brubeck 1956).

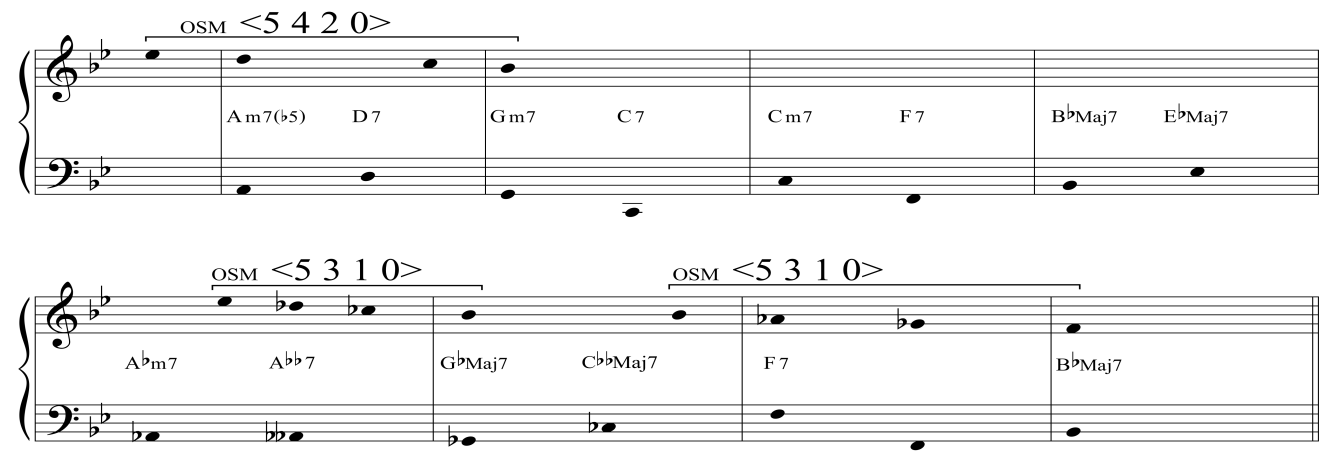

\footnotetext{
${ }^{9}$ Recordings of "In Your Own Sweet Way" by other artists—most notably Davis (1956) feature substitutions of $\mathrm{D} b 7$ for $\mathrm{A} b b 7$ at the fifth measure of each $\mathrm{A}$ section. This substitution creates 8-7-10 LIPs across mm. 1-2 and 5-6 that enhance the perceptibility of the related OSMs.

${ }^{10} \mathrm{E}$ natural, the other member of the inversional axis, does not sound in the melody in Brubeck's recording. However, Davis (1956)—which was the first commercially successful remake of the tune-features $E$ natural at the end of the A sections rather than F. In light of the symmetrical pattern shown in Figure 2, E natural happens to be the only pitch class that is not a member of either diatonic collection. It is also the only tone of the chromatic scale that does not function as a chord root in the A section. Subsequent recordings of "In Your Own Sweet Way" by many other artists include melodic E naturals at the ends of A sections. See, for example Montgomery (1960), Tyner (1976), Pass and Pedersen (1978), Remler (1982), Garrett (1995), and Hutcherson (1999). Gioia $(2012,197)$ also discusses the impact of this melodic change.
} 
Figure 2: Inversional symmetry established by ic4-related diatonic collections $\mathrm{Gm}$ and $\mathrm{G} b$ major.

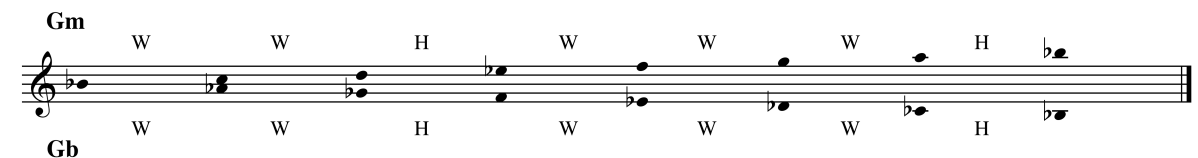

The final melodic gesture (mm. 6-8) capitalizes on the relationship between the two OSMs by recalling the general melodic rhythm of mm. 1-2 (not shown), placing its second and fourth pitches on downbeats and the first and third pitches within the beats that precede them. However, the occurrence of OSM $<5 \quad 3 \quad 10>$ as $\mathrm{B} b-\mathrm{A} b-\mathrm{G} b-\mathrm{F}$ in the final passage rather than a diatonically appropriate $\mathrm{OSM}<5420>$ as $\mathrm{B} b-\mathrm{A}-\mathrm{G}-\mathrm{F}$ produces a curious relationship between melody and harmony. Although harmonic motion leaves the local key of $\mathrm{G} b$ and cadences on $\mathrm{B} b$ at the end of the A section, the melody remains in the $\mathrm{G} b$ diatonic collection, and by doing so, it continues to evoke that key. ${ }^{11}$ By m. 8, it is possible to appreciate the harmonic arrival of the global tonic and the collection of tones it represents in light of the fact that melodic activity remains in the distantly related key established across $\mathrm{mm}$. 56. In this way, the A sections of this composition have harmonic closure while remaining melodically open to a considerable degree. Melodic closure is only attained at the final note, F, which is shared between the two keys but is more stable in $\mathrm{B} b$.

Granted, the tones required to keep the melody in $\mathrm{G} b$ while cadencing in $\mathrm{B} b$ amount to standard alterations (\#9 and b9) of a dominant harmony on F. The downward resolution through those ninths to the root of the dominant is idiomatic. Furthermore, one could argue that the downward resolution of a tone followed by a semitone creates a convincing arrival on any core chord tone (i.e., root, $3^{\text {rd }}, 5^{\text {th }}$, or $7^{\text {th }}$ ), even in the absence of dominant harmony. Another way to say this, however, would be that occurrences of OSM $<310>$ are idiomatic over harmonies when the final element of the motive is a core chord tone. And one possible way of describing the end of the A section of "In Your Own Sweet Way" observes that this idiomatic OSM is a subset of a motive that plays a larger role in this section. Moreover, the occurrence of OSM $<53$ $10>$ at the end of the A section is particularly effective in this composition, as it helps relate the key center of $\mathrm{G} b$ major (a tonality achieved by roaming perhaps too far afield in a series of falling fifth resolutions) with the global tonic.

11 This resonates in an interesting way with McFarland (2009), who discusses chordal polytonality in Dave Brubeck's compositions. 
Brubeck's use of OSMs to relate two distantly related key centers gives motivic depth to "In Your Own Sweet Way." Measures 5-8 of Example 4 show a similar passage from Horace Silver's "Peace," where the same family of diatonic OSMs occurs. ${ }^{12}$ OSM $<5310>$, an inversion of an initial diatonic OSM (mm. 1-2), sounds twice over ic4-related keys A major and $\mathrm{D} b$ major. Although the passage ends in D $b$, each OSM is a scale fragment from A major. The final three elements of the second occurrence sound again over $\mathrm{m}$. 7 , asserting the diatonic conflict by allowing both altered 9ths to sound over $A b 7$. The effect is much like that which occurs at the end of the A section of "In Your Own Sweet Way." The second key exerts the stronger influence, but this influence is distinctly colored by the melody's adherence to diatonic pitches from the immediately previous key. When this happens across the last two bars of "Peace," the subset OSM $<310>$ echoes the fragment that sounds over mm. 7-8, while conjoining the keys of $\mathrm{D} b$ major and $\mathrm{B} b$ major.

Example 4: Horace Silver, "Peace" (Silver 1959).
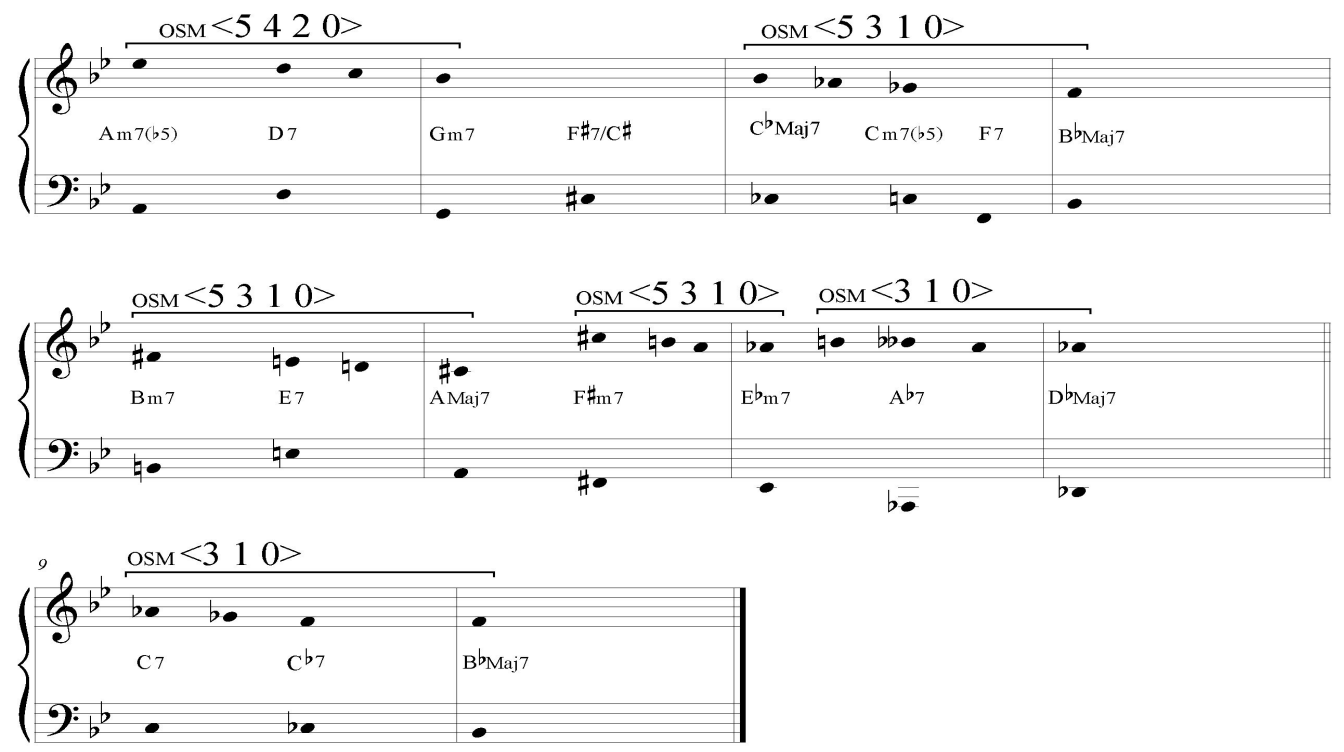

Figure 3: Inversional Symmetry around Bb in "Peace," mm. 1-4.

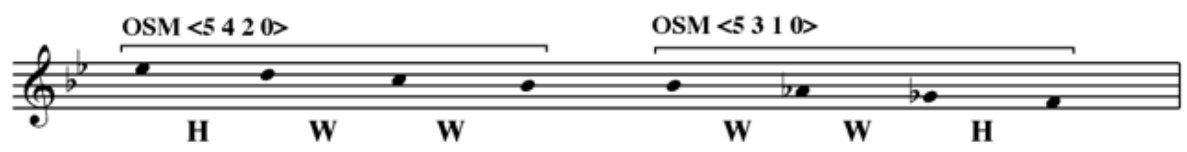

12 Note how the second occurrence of OSM $<5310>(\mathrm{mm} .5-7)$ relates strongly to the original OSM $<5420>(\mathrm{mm} .1-2)$ by incorporating the analogous scale degrees in $\mathrm{G}$ minor and A major, respectively. 
Further comparison of "In Your Own Sweet Way" and "Peace" reveals that both highlight the relationship between their initial motive, OSM $<542$ $0>$, and multiple occurrences of that motive's retrograde inversion, OSM $<53$ $10>$. Two factors invite us to appreciate this. Perhaps the most obvious is that these contrasted OSMs sound at the beginnings of successive phrases. No less important is the fact that elements within each OSM are comprised of the same sequence of local scale degrees, descending from submediant to mediant. The retrograde-inversional relationships come about through simple changes in modality; OSM <5 42 0> spans these degrees in minor, while OSM <5 31 $0>$ does so in major.

Finally, Examples 3 and 4 both demonstrate centricity around their global tonic, $\mathrm{B} b$, though they do so in different ways. OSMs related by retrograde inversion sound across the first phrase of Example 4, supported by the key centers $\mathrm{G}$ minor, $\mathrm{C} b$ major, and $\mathrm{B} b$ major. ${ }^{13}$ The confluence of these OSMs creates a symmetrical pattern of steps around the global tonic pitch, $\mathrm{B} b$, shown in Figure 3. In this way, "Peace" shows an even stronger correspondence to "In Your Own Sweet Way" (compare to Figure 2), where distantly related key centers create a similar arrangement of steps around a centric pitch class. The symmetry of "Peace," expressed by adjacent statements of related OSMs, is easier to hear.

The A section of "In Your Own Sweet Way" differs from the opening phrase of "Peace" in an important respect. While recurrent OSMs help us understand the relationship between two passages in distantly related keys in the former, a single OSM spans a distant modulation ( $\mathrm{C} b$ major $\rightarrow \mathrm{B} b$ major) across a single passage in the latter. In Examples 5-8, OSMs lend melodic continuity to other tonally adventuresome passages, often receiving contrapuntal support from LIPs. Example 5 is an analysis of Benny Golson's "Stablemates." ${ }^{14}$ In the A section of this composition, OSMs comprised of pitch classes from the global key of $\mathrm{D} b$ major function like a glue that bonds a number of tonal regions. The first two OSMs are tetrachords of the $\mathrm{D} b$ major scale that extend across harmonic activity in the tonalities of bII, I, IV, and iii, creating a complete statement of the $\mathrm{D} b$ major diatonic collection. OSM $<54$ $20>$ sounds across mm. 1-3, where ii $\rightarrow$ V7 motion in the neighboring key of

\footnotetext{
${ }^{13}$ Julien $(2001,54)$ discusses the nonfunctional nature of the harmonies in this passage.

14 The original recording of "Stablemates"-Davis (1955)—features no chordal accompaniment during its presentations of the melody (only drums, trumpet, and bass). Harmonies in Example 5 are implied by the bass line and clarified in the choruses of Davis's and Coltrane's solos, and confirmed by Chambers (1956) - recorded just four months later. See, however, the exception in footnote 18 .
} 
$\mathrm{E} b b$ major supports the initial pitch of the motive, and the final element involves resolution to the global tonic, $\mathrm{D} b .{ }^{15}$ The same motive sounds again across $\mathrm{mm}$. 7-9, where the tonics of local keys also relate by a descending semitone.

Example 5: Benny Golson, "Stablemates" (Davis 1955).
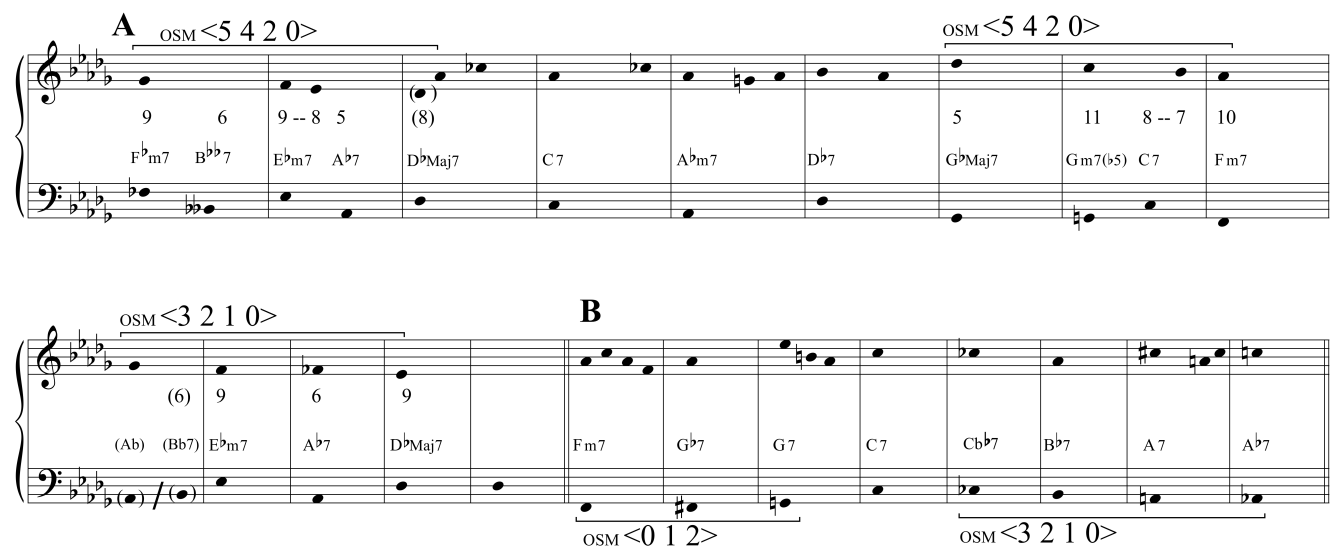

A common variation of a standard LIP underlies the first occurrence of this motive. The sequence of intervals against the bass, 9-6-9-8-5-8 demonstrates the relationship between 9-6 and 8-5 LIPs. Strunk (1996, 8689) notes that the 9-8 suspensions may embellish 8-5 patterns, and that in some cases ninths can carry over changes of bass and become sixths. ${ }^{16}$ At the opening of this composition, neither ninth nor sixth resolve. The occurrence of this somewhat less conventional 9-6 pattern coincides with the implication of the distantly related key of $b \mathrm{II}(\mathrm{E} b b)$. Only when harmonic motion becomes diatonic with respect to the global key at $\mathrm{m}$. 2 does the ninth resolve to an octave and the more conventional 8-5-8 LIP sound.

When OSM $<5420>$ sounds again across $\mathrm{mm}$. 7-9, tonicizations of $\mathrm{G} b$ major and $\mathrm{F}$ minor occur in succession. Here, the sequence 5-11-8-7-10 sounds, beginning with a somewhat unconventional series of intervals but

${ }^{15} \mathrm{I}$ interpolate the implied tone $\mathrm{D} b$ at $\mathrm{m} .3$ for the following reasons: It is the root of the harmony at this measure, which is the local and global tonic. The melodic leap from Eb4 to $\mathrm{Cb} 5$ at the end of m. 2 (not shown) clearly implies motion to another voice, and it does not influence motion within the specific voice where the OSM occurs. This argument resonates with that which supports the implied tones in Example 1 of Rothstein (1991, 289-292).

${ }^{16}$ Strunk (1996) prefers "thirteenths" to "sixths." I prefer the smaller number because the focus of this study concerns outer-voice counterpoint and not extended harmony, and because it more clearly represents the displacement of the fifth of a normative LIP by a step. 
terminating with a well-worn 7-10 pattern. ${ }^{17}$ This occurrence of OSM $<542$ $0>$ helps the passage relate to the rest of the composition by using pitch classes from the global tonic scale, traversing an interval of the tonic triad $(\mathrm{D} b-\mathrm{C}-$ $\mathrm{B} b-\mathrm{A} b)$, and featuring the same OSM that sounds over the establishment of tonic across mm. 1-3.

Example 5 also shows how the chromatic motive OSM $<3210>$ sounds at the end of the A section, supported by the LIP 9-6-9. This motive begins like the initial occurrence of OSM $<542$ 0>, with motion from Gb4 to F4. However, it continues to move one half step at a time and only arrives on the ninth of the tonic chord by the end of the melody. The structure and setting of OSM <3 2 1 $0>$ across mm. 10-13, a chromatic motive supported by conventional diatonic progression (ii $\rightarrow \mathrm{V} 7 \rightarrow \mathrm{I} 7$ ) in a single key, are quite the opposites of those that characterize occurrences of OSM $<5420>$, which always express a diatonic scale segment while sounding against harmonic movement that modulates. ${ }^{18}$

Much of the harmonic motion across the bridge of "Stablemates" resonates with the OSM that closes the A section. Example 5 illustrates how OSM <3 21 0> describes root movement across mm. 19-22. Perhaps less noticeably, root movement across mm. 15-17 (Fm7 $\rightarrow \mathrm{Gb} 7 \rightarrow \mathrm{G} 7)$ projects OSM $<012>$, which is a subset of what we might perceive as either the retrograde or the inversion of OSM $<\begin{array}{lllll}3 & 2 & 1 & 0>. & .\end{array}$ connection less obvious and possibly irrelevant, but the occurrence of OSM $<0$ $12>$ is significant for several reasons: It sounds immediately after the original OSM $<3210>$, with pitches sounding at the same rate of one per measure. Its first two pitch classes, $F$ and $G b$, are the same as those in the initial occurrences of OSM $<3210>$ and $<5420$. Finally, the OSM sounds across a passage where changing harmonies underpin a melodically persistent $\mathrm{A} b 4$ that is embellished by leaps. A similar process occurs beneath $\mathrm{A} b 4$ across mm.

${ }^{17}$ We may regard this unconventional series of intervals as the result of a misalignment of voices. In this case, the melody changes ahead of the bass. If $\mathrm{D} b 5$ continued to sound across the first half of $\mathrm{m} .8$, and subsequent motion through $\mathrm{C} 5$ to $\mathrm{Bb} 4$ sounded over the second half, the resultant LIP (5-8-7-10) would be less unusual.

${ }^{18}$ During presentations of the melody, bassist Paul Chambers plays $\mathrm{A} b 2$ at the downbeat of the tenth measure of each A section and rests for the remainder of that measure. By implying $\mathrm{V}$ here, the original recording highlights those contrasts of chromatic and diatonic harmony and melody described above. However, $\mathrm{B} b 7 \# 9$ is the operative harmony at this spot during solos. It also sounds at every instance in Chambers (1956), recorded just four months later, as well as in releases by the composer himself, such as Golson (1997). The addition of V7/ii in these cases detracts from the contrast, but extends the LIP by creating a sixth below Gb4

${ }^{19}$ As the step patterning of this motive is symmetrical, either perception is possible. 
3-6. And, as mm. 4-6 constitute the only sizable span of the A section where no OSMs sound, the occurrence of a comparable melodic event in the bridge with OSM patterning makes a large-scale contribution to compositional design.

Most of the OSMs in the analyses above feature diatonic scale segments. Taken at face value, this statement should not be surprising. After all, diatonic collections are eminently familiar, and tonal melodies often employ conjunct, linear fragments of them. The diatonic OSM that sounds across mm. 7-9 of "Stablemates" is particularly unifying, as its constituent pitch classes are subsets of the two key centers that underlie the passage ( $\mathrm{Gb}$ and Fm), and of the global tonic as well. Even OSMs that belong to diatonic collections that are different from the tonalities conveyed by their supporting harmonies (such as mm. 6-8 of Example 3 and mm. 6-7 of Example 4) are not unusual in standard jazz. Although OSM $<4310>$, the primary motive in the next two compositions, is not a diatonic scale fragment, it is still familiar to listeners of tonal music. It may span $\wedge 3$ and $\wedge 7$ of any minor mode with a leading tone. Or it may arise in cases of modal mixture, as when $b^{\wedge} 6-\wedge 5-\wedge 4-\wedge 3$ sounds in a major key. OSM $<4310>$ has a special place in the melodic language of jazz. To understand this motive's role in jazz composition, it is necessary to know at least a small part of its history. OSM $\langle 4310>$ is common in improvisationparticularly in those cases of modal mixture where a 9-8-7-10 LIP sounds over the course of an authentic cadence. One needs only to listen to a reasonable sampling of recordings from innovative jazz players between 1940 and 1954 to appreciate the popularity of this motive during this era. ${ }^{20}$

As popular as this motive was among jazz musicians, our next example was written by a Broadway composer in 1939 . The song "All the Things You Are" came from a little known musical, and is the only jazz standard to have emerged from it. Forte $(1995,73)$ attributes the success of the tune to "subsequent generations of musicians, especially ... jazz performers, and arrangers and singers as well." This is undoubtedly due in part to the fact that the song's structure resonates deeply with how jazz musicians appreciate harmony, melody, and overall form. Example 6 shows how OSMs delineated by LIPs extend across mm. 1-24. Abrupt modulations across mm. 5-8 and 13-16 punctuate eight-measure phrases comprised entirely of 10-7 LIPs.

\footnotetext{
${ }^{20}$ For examples of improvised occurrences-some of which are embellished-see Clifford Brown's solo on "Split Kick" from Blakey (1954), Don Byas' solo on "Back Home Again in Indiana" from Byas and Stewart (1945), or Dexter Gordon's solo on "Setting the Pace" from Gordon (1947). Also note its later occurrence in a compositional context across mm. 9-11 of "It’s A Raggy Waltz" (B b-A-G-F\#, Eb-D-C-B) in Brubeck (1961).
} 
These modulations help create OSM $<4310>$ across each phrase, with each OSM element initially supported as a $10^{\text {th }} .{ }^{21}$ Given that the two phrases across mm. 1-16 are nearly exact transpositions of each other, we should expect motivic recurrences. However, one significant difference between the two phrases involves the more local occurrence of OSM $<4310>$ across mm. 1415 that retraces the descent from Eb that began at m. 9.22 10-7-10 LIPs across the bridge create occurrences of OSM $<310>$ in the distantly related keys of $\mathrm{G}$ major and $\mathrm{E}$ major. ${ }^{23}$ The pitches of these motives align in such a way to create OSM <6 4310 , a superset of the original motive that continues the pattern of alternating tones and semitones. ${ }^{24} \mathrm{OSM}$ in this composition are remarkable for at least two reasons. All of them are formed through the use of $10-7$ or 10-7-10 LIPs. More relevant to this study, however, is how several of the OSMs incorporate abrupt modulations between distantly related keys at their conclusions.

Example 6: Jerome Kern, "All the Things You Are," mm. 1-24 (Kern and Hammerstein II 1939).
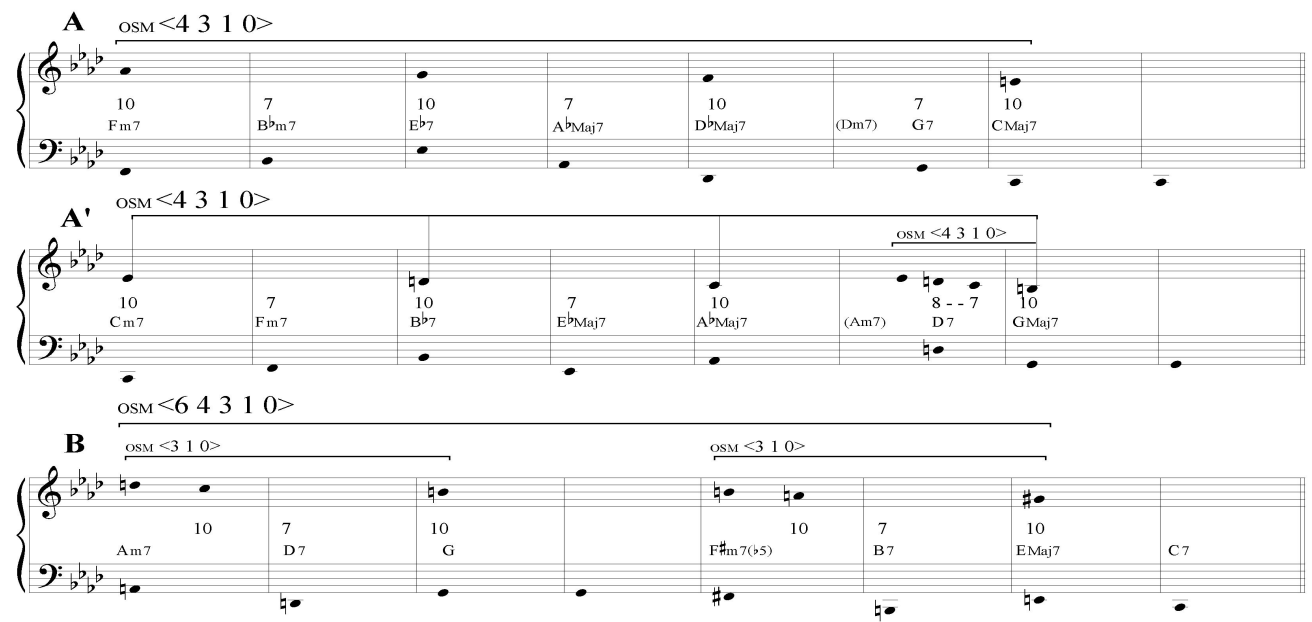

Example 7 shows Dizzy Gillespie's "Con Alma," a 32-bar composition in AABA form described in Waters $(2011,27)$ as among "such works that create

${ }^{21}$ In discussing one of these modulations, Forte $(1995,76)$ remarks: "Although this change... does not affect the interval pattern $10-7$, it does bring about a significant harmonic reorientation."

${ }^{22}$ Larson $(1998,237)$ offers the term "confirmation" to account for the phenomenon of a melodic line reiterating the complete path of its structural descent at its conclusion.

${ }^{23}$ See Forte $(1995,76)$ for a discussion of the difference between 10-7 and 10-7-10 patterns.

${ }^{24}$ Forte $(1995,76-79)$ discusses LIPs in this song, as well as the melodic connection between bridge and the final A section. Forte's Example 74 (p. 79) is a reduction that corresponds considerably to my own Example 6. 
localized key areas but avoid a clear single overall tonic.” Strunk $(2002,168)$ discusses "Con Alma" as an example of progressive tonality in jazz composition. In reference to a group of tonally ambiguous jazz compositions, he observes "Consideration of melodic and voice-leading aspects ... would be necessary for a better analytical understanding of their structure." OSM analysis answers this call in part by revealing connections between the melodic surface of this composition and its underlying harmonic organization. Nested occurrences of OSM <4 31 0> sound across the A section. Both four-bar phrases end with the motive, and parallel tenths formed by the local tonic major seventh chords that begin and end each phrase create an occurrence that spans the whole section. And, in a manner that recalls mm. 9-17 of "All the Things You Are," the last four notes of the melody at m. 8 (Ab-G-F-E) retrace the path of the large-scale descent that crosses the section.

Example 7: Dizzy Gillespie, "Con Alma" (Gillespie 1954).
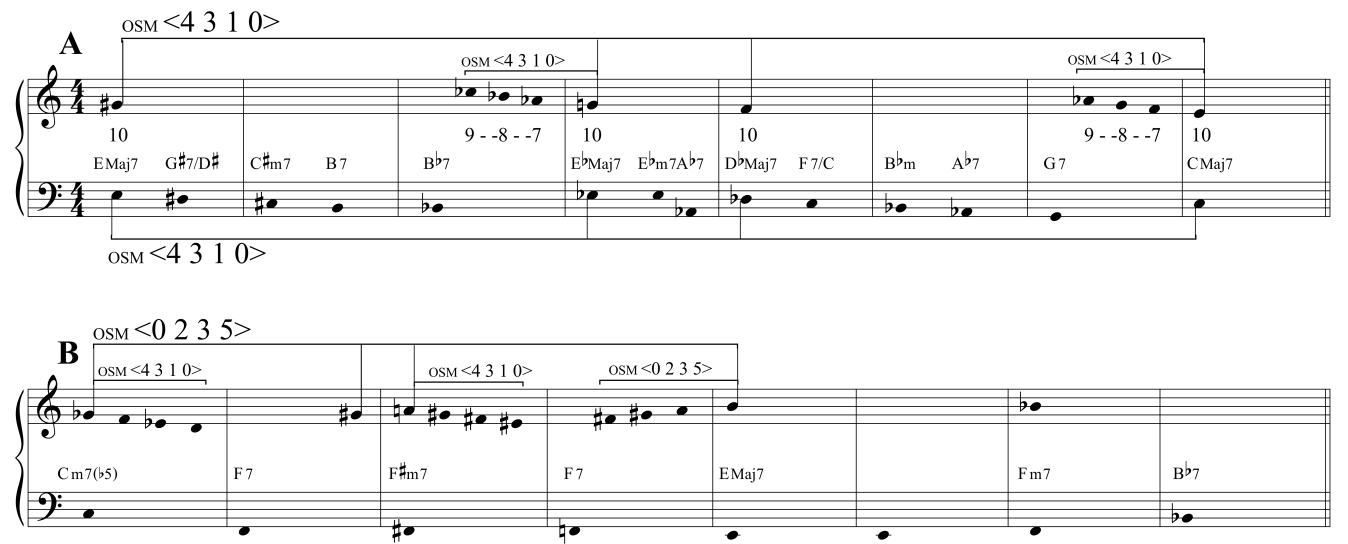

Although the smaller occurrences of OSM $<4310>$ do not span changes of key, Gillespie's use of the motive at multiple levels of structure is still quite innovative. The motive's popularity as a jazz lick through the 1940s and early 1950s, suggests that it was conventional enough within the melodic language of jazz by 1954, when this piece was written. At this point, OSM <4 $310>$ could serve as a signifier that enables listeners to follow the unusual modulations of this piece and also accept (or be comfortably diverted from) the fact that the piece lacks a global tonic. So, while local occurrences of OSM $<4$ $310>$ do not span modulations, the composition takes advantage of the referential function of OSM $<4310>$ at local and global levels of structure.

Example 7 also shows how OSM $<0235>$ sounds across the B section. $\mathrm{G} b 4$ and A4, both registrally prominent and metrically emphasized pitches, trace the general upward thrust of the melody across mm. 17-24 with G\# 
connecting. B4 (mm. 21-22) is the melodic goal of the whole section, enjoying metric, agogic, and registral emphasis. Smaller-scale OSMs also occur across this passage, and they do so in ways that help the $\mathrm{B}$ section correspond to the A sections. First, OSM <4 $310>$ sounds twice across mm. 17-20 in sequence, and these occurrences invite comparison to mm. $1-8 .^{25}$ In addition, a pitchspecific hidden repetition of the large-scale OSM <0 $235>$ aligns with the arrival of $\mathrm{B} 4$ at $\mathrm{m} .21$, echoing the nested motive that occurs at the end of the A section. From an intervallic perspective, OSM $<\begin{array}{llll}0 & 2 & 3 & 5\end{array}>$ represents a 'turning inside-out' of OSM <4 $310>$. That is, whole steps occur in the former where half steps do in the latter, and vice-versa. The change in direction is also important. Bridges often feature OSMs that travel in opposite directions from those of A sections. Recall, for instance, the inversional relationships between OSMs in Examples 1 and 5. ${ }^{26}$

Example 8: Large-scale OSMs in "Con Alma."

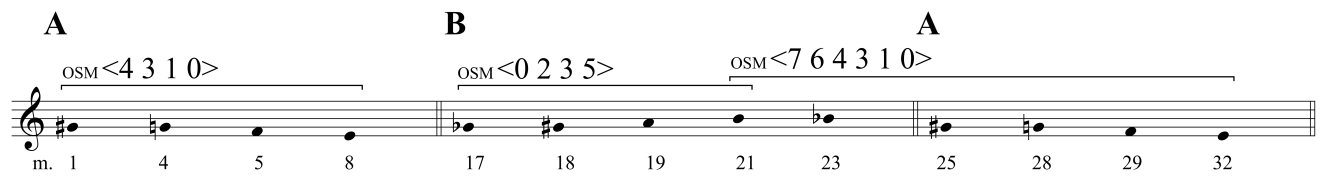

OSMs also help us account for the rather unconventional harmonic connection that occurs at the end of the bridge. Here, melodic motion from B4 to $\mathrm{B} b 4$ across $\mathrm{mm}$. 21-24 sounds above a transition from a tonic $\mathrm{E}$ major to a II7 $\rightarrow \mathrm{V} 7$ progression in $\mathrm{E} b$ major. While it certainly is not unusual for bridges to conclude with dominant chords, the dominant in this case does not align with the $\mathrm{E}$ major tonic chord that sounds at the beginning of the $\mathrm{A}$ section that follows. In other words, the occurrence of a II7 $\rightarrow \mathrm{V} 7$ progression in $\mathrm{E} b$ major between two $\mathrm{E}$ major tonics is atypical, even by the conventions of mid-twentieth century modern jazz. Example 8 shows that the melodic $\mathrm{Bb}$ supported by the progression creates an important link between the apex of the bridge and the following $A$ section. It does so by initiating the descending pattern of alternating whole steps and half steps characteristic of the A section's OSM <4 31 0> several measures in advance of m. 25. Furthermore,

${ }^{25}$ Many recorded versions of this tune feature both occurrences of OSM $<4310>$ here, although some performers will play $\mathrm{E}$ natural instead of $\mathrm{E} \#$ in the third measure of the bridge. Several of Gillespie's own recorded performances feature both occurrences of OSM $<4310$, including the original, Gillespie (1954). See also Gillespie (1997), as well as Peterson and Gillespie (1974).

${ }^{26}$ Inversionally related OSMs also extend across different sections of "Autumn Leaves," a harmonically and tonally unambiguous song. OSM $<5420>$ sounds across mm. 1-8 of the A section ( $\left.b^{\wedge} 6-^{\wedge} 5-\wedge 4-b^{\wedge} 3\right)$, while OSM $<0135>$ sounds across the first 8 measures of the $B$ section $(\wedge 2-b \wedge 3-\wedge 4-\wedge 5)$. 
the general rate at which the bridge's elements of this large-scale OSM occur (that is, two elements every four measures) is consistent with the general rate at which elements occur in the A section. The result is superset OSM <7 6431 $0>$, what one could regard as two overlapping occurrences of OSM $<4310>$. In this way, a large-scale OSM reveals continuity in a part of the composition that is harmonically peculiar.

Example 9: Herbie Hancock, "Dolphin Dance," mm. 1-24 (Hancock 1965).

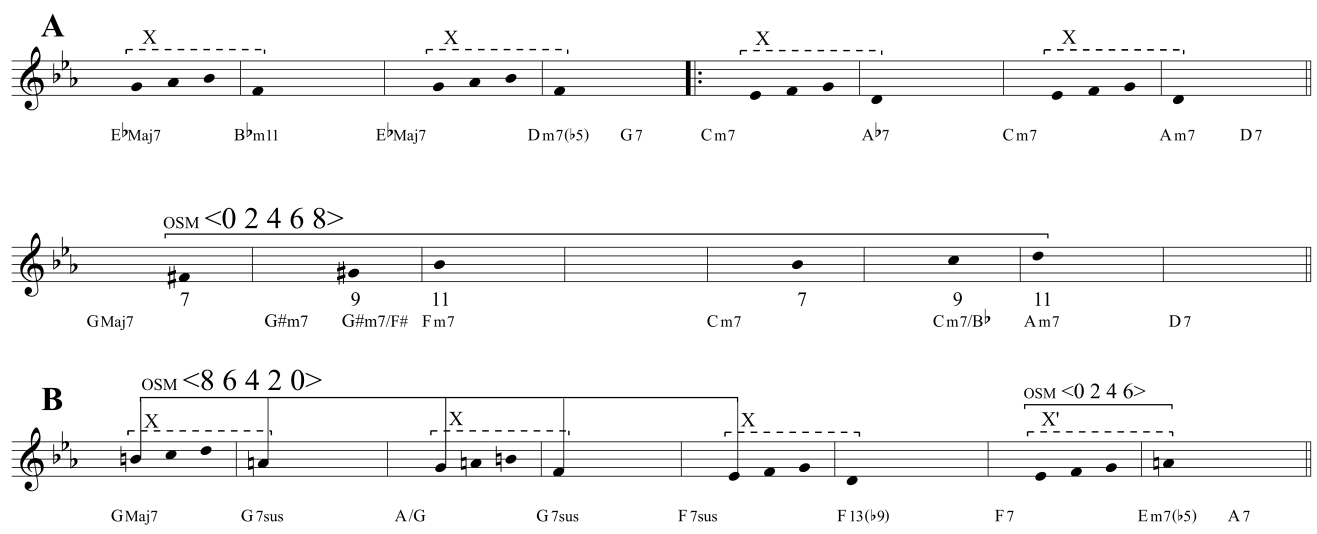

The last two analyses of this study deal with OSMs in tonally ambiguous works with nonfunctional harmonic connections. ${ }^{27}$ Example 9 shows mm. 124 of Herbie Hancock's "Dolphin Dance." Here, OSMs are subsets of the whole-tone collection that interact with a recurrent surface melodic motive (labeled "X"). ${ }^{28}$ OSM $<02468>$ sounds across the last eight measures of the A section, created by a harmonic and melodic sequence that produces 7-9-11 LIPs. In reciprocity, OSM $<86420>$ sounds across the first five measures of the B section. ${ }^{29}$ Here, as in "It's A Raggy Waltz," "Stablemates," and "Con Alma," correspondences between ascending and descending OSMs provide a degree of unity between two contrasting sections. In "Dolphin Dance," a significant difference between sections $\mathrm{A}$ and $\mathrm{B}$ is harmonic. The longsounding pedal tones across mm. 17-24 render $\mathrm{B}$ less active than $\mathrm{A}$ in this

${ }^{27}$ Certain analyses by Steven Strunk and Keith Waters acknowledge the interaction of linear motives with nonfunctional harmony in composition. See Strunk (2005, 321-322), which discusses Wayne Shorter's "Night Dreamer," and Waters (2005, 350-352) and (2011, 229), which discuss "Dolphin Dance" and "Pinocchio," respectively.

${ }^{28}$ See also Waters $(2010,150-154)$, which discusses how much of the same melodic material featured in this study interacts with ic4-related "regions" (akin to key areas). The presence of ic4 relations and whole-tone subsets in this composition is certainly not coincidental.

${ }^{29}$ This analysis observes the formal divisions put forth in Waters $(2005,350)$, with A spanning mm. 1-16 and B, mm. 17-24. 
respect. Melodically, B summarizes motivic activity from A, as surface recurrences of the ubiquitous $X$ motive from the first half of $A$ embellish a variation of the subsurface whole-tone motive from the second half. ${ }^{30}$ The occurrence of OSM $<0 \quad 24$ 6> across mm. 23-24 punctuates this correspondence. It consists of the last four pitches of OSM $<86420>$ in reverse, and it sounds in rhythmic correspondence to the $\mathrm{X}$ motive introduced in the A section.

My final analysis reveals how connections between surface-level motives and those that lie beneath them can influence our perception of continuity in a composition with a circular form. A circular composition will not have a conclusive ending, so that upon repetition, the recurrence of the top of the form will sound like a seamless continuation. Example 10 is an analysis of Wayne Shorter's "Nefertiti," a work characterized by non-functional harmonic connections, ambiguous tonality (Strunk 2002, 168; Waters 2011, 219), as well as circular form (Larson et al. 2009; Waters 2011, 217-219). ${ }^{31}$ The initial phrase, mm. 1-8, presents all of the relevant motivic material. Here, two families of three-element OSMs emerge. OSM <2 $10>$ and OSM < $220>$ sound as the melody descends. ${ }^{32}$ Their retrograde counterparts, OSM $<024>$ and OSM $<012>$, answer them as the melody ascends. ${ }^{33}$

Example 10: Wayne Shorter, "Nefertiti" (Davis 1967).

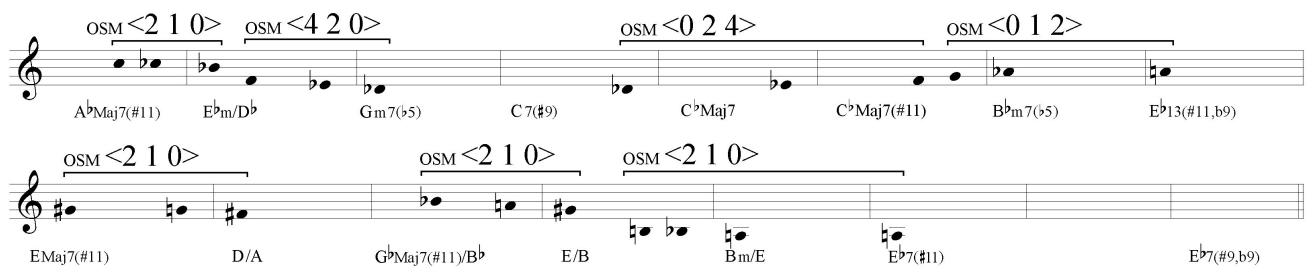

\footnotetext{
${ }^{30}$ In addition to this correspondence, Waters (2005, 352) observes motivic connections between the bass in the $\mathrm{B}$ section through the start of the $\mathrm{A}$ section $(\mathrm{G}-\mathrm{F}-\mathrm{E} b)$ and middleground-level melodic material that occurs earlier. "We may hear this descending third motive in the bass between $\mathrm{mm}$. 17 and 25 as motivated by the descending third motive that appears in the opening melody between $\mathrm{mm} .1-5 . "$

${ }^{31}$ The chord symbols in Example 10 correspond to Example 6.4a in Waters $(2011,218)$.

${ }^{32}$ In Shorter's melody, $\mathrm{C} 4$ sounds between $\mathrm{F} 4$ and $\mathrm{E} b 4$ at $\mathrm{m}$. 2. This analysis regards the downward leap of a fourth and subsequent upward leap of a third as motion to and away from another voice- a voice that creates tension against the sounding harmony but resolves upward to $\mathrm{D} b 4$ in the next measure.

${ }^{33}$ Since both OSMs are symmetrical, inversionally related forms are identical to retrograde forms. Since the occurrences of OSMs $\langle 420>$ and $\langle 024>$ involve the same pitches, the more intuitive operation is retrograde. By extension of this association, I consider OSMs $<210>$ and $\langle 012\rangle$ in this phrase retrograde related as well.
} 
Both of these families of OSMs play roles at deeper structural levels, and these motivic recurrences can affect how we perceive the circularity of form in this composition. Strunk (in Larson et al. 2009) comments on circular form in general, observing two melodic characteristics that contribute to circularity.

- stopping on a note not tonally at rest

- not stopping, leading directionally back to the beginning

Waters (2011, 75) enumerates three conditions for circularity (melodic, harmonic, and hypermetric) in compositions written by the second Miles Davis Quintet. Only the first of these is directly relevant to this composition, and it is essentially a more specific description of Strunk's second characteristic.

- melody: the opening phrase of the composition sounds as a continuation of the previous phrase

Strunk's characteristics would seem mutually exclusive, but "Nefertiti" actually exhibits both of them at different levels of structure. As the final melodic pitch, A natural, is dissonant with respect to the harmony over which it sounds (Eb7), as well as to what is arguably the most viable candidate for a global tonic (Ab major), we can easily hear how "Nefertiti" exhibits Strunk's first characteristic. ${ }^{34}$ From a broader perspective, however, OSMs connect the end of the melody with its beginning, and in this way, "Nefertiti" exhibits Strunk's second characteristic. In other words, when we understand the role of the A natural with respect to the interplay of OSMs, we can hear a melodic connection between m. 14 and the downbeat of m. 1. Waters $(2011,217)$ observes how the pitches that initiate melodic descents at $\mathrm{mm} .9$ and 11 create a large-scale stepwise ascent that connects to the beginning of the first phrase as the form repeats $(\mathrm{G \#} \rightarrow \mathrm{B} b \rightarrow \| \mathrm{C})$. Example 11 recognizes this ascent as a large-scale statement of OSM $<02$ 4>. Example 10 shows how OSM <2 10 > sounds at the beginnings of each of these gestures, while Example 11 transposes the occurrence of OSM $<210>$ across mm. 12-13 (B-B b-A) up one octave. Example 11 reveals how this motive unites the two OSM families by filling in a large-scale $<012>$ motive that is nested within the larger statement of OSM $<024>$. The relationship between this $<012>$ motive and the OSM $<210>$ at $\mathrm{m} .1$ as linked retrogrades $(\mathrm{B} b-\mathrm{B}-\mathrm{C}-\mathrm{C} b-\mathrm{B} b)$ contributes to the effectiveness of the circular formal design.

\footnotetext{
${ }^{34}$ Waters $(2011,219)$ observes the "larger sense of $\mathrm{Ab}$ tonality suggested by the opening $\mathrm{Ab}$ harmony and the Eb7 harmonies that close phrases 1 and 3."
} 
Example 11: Large-scale OSMs in "Nefertiti."

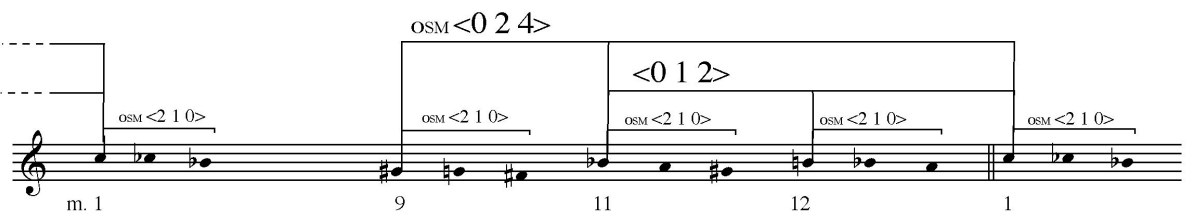

The transposition of elements within an OSM by one or more octaves generalizes that motive to the realm of abstraction involving pitch class. According to the definition of an OSM at the beginning of this article, the linear $<012>$ motive in Example 11 is not even an OSM. The local OSM at m. 12 does play an interesting role at the pitch level where it sounds. At the melodic surface, the occurrence of two descending semitones in a lower octave allows us to hear that the melody is trying to come to its close. This register provides a reasonable contrast with the end of the first phrase, which terminates on the same tone an octave higher. However, at deeper levels of structure-a depth commensurate with those levels of tonal music where stepwise connections between pitch classes often begin to arise-OSM $<210>$ interacts with and connects to the other forms of the motive that sound in its temporal environment. What may we call the resultant mid-level $<0 \quad 12>$ motive? How may we classify it? How should we limit it? While the answers to these questions are outside this article's scope, it is no small consolation that by exceeding the limits of the OSM, we reveal further possibilities for meaning in music - for that is perhaps the best way to conclude an analytical study.

\section{CONCLUSION}

The examples in this study do not support any claim that the melodic necessarily precedes the harmonic when composers create tonally adventuresome or formally ambiguous works. Rather, they reveal how linear melodic connections, as well as those in other voices, can help us make sense of the more unusual harmonic turns that compositions can take. Meyer (1989, 46) makes a resonant observation in an essay on style analysis that is also careful not to prioritize melody or harmony. In a description of Wagner's use of motives, he remarks: "Sequential organization-particularly when motives are intervallically fixed or when harmonization is a characteristic feature of the motive-easily leads to chromaticism and, through this, to modulation." Of course, as OSM modeling recognizes variants under canonic operations as well as subset/superset relationships, it necessarily involves a more generalized sort of motivic equivalence than Meyer had in mind. But this generalization is necessary to reveal some of the characteristics of standard jazz repertoire. Still, 
in light of Meyer's insight, we may predict that OSM analysis-or some adaptation thereof that incorporates strict intervallic correspondence with changes in melodic contour-could describe a great deal of chromatic tonal music from a number of different idioms.

\section{REFERENCES}

Baker, James. 1980. “Scriabin's Implicit Tonality.” Music Theory Spectrum 2/1: 1-18.

Forte, Allen. 1995. The American Popular Ballad of the Golden Era 1924-1950. Princeton University Press.

Friedmann, Michael. 1985. "A Methodology for the Discussion of Contour: Its Application to Schoenberg's Music.” Journal of Music Theory 29/2: 223-248.

Gioia, Ted. 2012. The Jazz Standards: A Guide to the Repertoire. Oxford University Press.

Joplin, Scott. 1909. Solace. New York: Seminary Music.

Julien, Patricia 2001. "The Function of Non-Functional Harmony." Jazz Educators Journal 34/2: 52-56.

Kern, Jerome and Oscar Hammerstein II. 1939. All the Things You Are. (From "Very Warm for May") Lyrics by Oscar Hammerstein II, Music by Jerome Kern. Copyright (C) 1939 PolyGram International Publishing, Inc.

Larson, Steve. 1993. "Dave McKenna's Performance of 'Have You Met Miss Jones.” American Music 11/3: 283-315. . 1998. "Schenkerian Analysis of Modern Jazz: Questions About Method." Music Theory Spectrum 20/2: 209-241. . 1999. "Swing and Motive in Three Performances by Oscar Peterson." Journal

of Music Theory 43/2: 283-314. . 2003. "What Makes a Good Bridge?" Tijdschrift voor muziektheorie 8/1: 1-15. 2005. “Composition versus Improvisation?” Journal of Music Theory 49/2: 241-275.

. 2009. Analyzing Jazz: A Schenkerian Approach. Hillsdale, NY: Pendragon Press.

Larson, Steve, Henry Martin, Steven Strunk, and Keith Waters. 2009. Circular Thinking: A Roundtable on "Blue and Green" and "Nefertiti." Paper presented at the annual meeting of the Society for Music Theory in Montréal, Quebec.

McFarland, Mark. 2009. “Dave Brubeck and Polytonal Jazz.” Jazz Perspectives 3/2: 153-176.

Martin, Henry. 1996 Charlie Parker and Thematic Improvisation. Lanham MD: Scarecrow Press. 
2005. "Balancing Composition and Improvisation in James P. Johnson's 'Carolina Shout.” Journal of Music Theory 49/2: 277-299. . 2006. "We Remember Clifford: Variation Technique at the Middleground." Tijdschrift voor muziektheorie 11/1: 1-8.

. 2011. "Schenker and the Tonal Jazz Repertory." Tijdschrift voor muziektheorie 16/1: 1-20.

Marvin, Elizabeth and Paul Laprade. 1987. "Relating Musical Contours: Extensions for a Theory of Contour." Journal of Music Theory 31/2: 225-267.

Meyer, Leonard B. 1989. Style and Music: Theory, History, and Ideology. University of Chicago Press.

Morgan, Robert P. 1976. "Dissonant Prolongation: Theoretical and Compositional Precedents." Journal of Music Theory 20/1: 49-91.

Morris, Robert. 1987. Composition With Pitch Classes: A Theory of Compositional Design. New Haven: Yale University Press.

Pressing, Jeff. 1978. “Towards an Understanding of Scales in Jazz.” Jazzforschung/Jazz Research 9: 25-35.

Rothstein, William. 1991. “On Implied Tones.” Music Analysis 10/3: 289-328.

Salzer, Felix. 1962. Structural Hearing. New York: Dover Publications.

Strunk, Steven. 1985. "Bebop Melodic Lines: Tonal Characteristics." Annual Review of Jazz Studies 3: 97-120.

. 1996. "Linear Intervallic Patterns in Jazz Repertory." Annual Review of Jazz Studies 8: 63-115.

. 2002. Harmony. The New Grove Dictionary of Jazz. $2^{\text {nd }}$ ed. London: Macmillan.

. 2005. "Notes on Harmony in Wayne Shorter's Compositions 1964-1967." Journal of Music Theory 49/2: 301-332.

Tymoczko, Dmitri. 1997. "The Consecutive-Semitone Constraint on Scalar Structure: A Link Between Impressionism and Jazz.” Intégral 11: 135-179.

Waters, Keith. 2005. "Modes, Scales, Functional Harmony, and Nonfunctional Harmony in the Compositions of Herbie Hancock." Journal of Music Theory 49/2: 333-357.

. 2010. “Giant Steps and the ic4 Legacy.” Intégral 24: 135-162.

2011. The Studio Recordings of the Miles Davis Quintet 1965-68. New York: Oxford University Press.

RECORDINGS

Blakey, Art. 1954. A Night At Birdland With Art Blakey Quintet, Vol. 1. Blue Note BLP 1521. 
Brubeck, Dave. 1956. Brubeck Plays Brubeck. Columbia CL 878. . 1961. Time Further Out. Columbia CL 1690.

Byas, Don and Slam Stewart. 1945. Indiana. Commodore 6.24058.

Chambers, Paul. 1956. Chamber's Music. Imperial LP 9182.

Davis, Miles. 1955. The New Miles Davis Quintet. Prestige LP 7014. . 1956. Workin' With The Miles Davis Quintet. Prestige PRLP 7166. 1967. Nefertiti. Columbia CS 9594.

Garrett, Kenny. 1995. Triology. Warner Bros. 9 45731-2.

Gillespie, Dizzy. 1945. Musicraft 488. .1954. Afro. Norgran MGN 1003, Verve MGV 8191. 1957. Birks Works: Dizzy Gillespie and His Orchestra. Verve MGV 8017. 1997. Bird Songs: The Final Recordings. Telarc TLC 83421.

Golson, Benny. 1997. Up Jumped Benny. Arkadia Jazz 70741.

Gordon, Dexter. 1947. Sett'n the Pace. Savoy/Arista LP2211.

Hancock, Herbie. 1965. Maiden Voyage. Blue Note BLP 4195.

Hutcherson, Bobby. 1999. Little B's Poem. e. j.'s music CD 614 (recorded in 1980).

Monk, Thelonious. 1951. Thelonious Monk Quintet. Blue Note BLP 1589.

Montgomery, Wes. 1960. The Incredible Jazz Guitar of Wes Montgomery. Riverside RLP 12-320.

Pass, Joe and Niels-Henning Ørsted Pedersen. 1978. Chops. Pablo 2310-830.

Peterson, Oscar and Dizzie Gilelspie. 1975. Oscar Peterson and Dizzie Gilelspie. Pablo 2310-740.

Remler, Emily. 1982. Take Two. Concord CJ-195.

Silver, Horace. 1959. Horace Silver Quintet. Blue Note BLP 4017.

Tyner, McCoy. 1976. Chick Corea, Herbie Hancock, Keith Jarrett, and McCoy Tyner. Atlantic 1696-2.

\section{ABOUT THE CONTRIBUTOR}

KEITH SALLEY received his BM in jazz guitar performance from the University of Memphis and his MA in music theory from Tulane University, where he wrote a thesis on harmonic substitution in jazz. He holds a doctorate in music theory with a cognate in jazz performance from the University of Oregon. Today, he is an Associate Professor of music and coordinator of music theory at the Shenandoah Conservatory in Winchester, VA. 